\title{
HIGHLIGHTS
}

REPRODUCTIVE ENDOCRINOLOGY

\section{High risk of liver disease in women with polycystic ovary syndrome}

One in four women with polycystic ovary syndrome (PCOS) may have nonalcoholic steatohepatitis (NASH), the most severe form of nonalcoholic fatty liver disease (NAFLD), according to an article published in the Journal of Clinical Endocrinology and Metabolism. Moreover, measurement of liver enzyme levels might fail to spot such liver damage.

NAFLD, which is associated with insulin resistance and the metabolic syndrome, is the most common type of liver disease. Insulin resistance is a key feature of PCOS and, as a consequence, women with the syndrome have an increased risk of NAFLD. Although NAFLD does not necessarily affect liver function, progression to NASH markedly increases the risk of cirrhosis. The prevalence of NASH among women with PCOS is difficult to determine because liver biopsy, an invasive procedure, is the only definitive way to distinguish NASH from other forms of liver disease, although persistently elevated levels of liver enzymes (alanine aminotransferase [ALT] and aspartate aminotransferase [AST]) can be used to indicate hepatocyte damage and the need for diagnostic liver biopsy tests.

To sidestep this difficulty, Tan et al. evaluated the prevalance of NASH in women with PCOS by the use of a novel noninvasive diagnostic test for NASH. The test detects the enhanced hepatocyte-cell death that accompanies NASH. Levels of a particular cleaved fragment of cytokeratin 18 (CK-18), produced during an apoptotic cell death pathway in the liver, are measured by a specific enzymelinked immunosorbent assay kit.

The researchers measured CK-18 fragment levels of 192 currently untreated women with PCOS and 73 age-matched healthy women. Metabolic factors and levels of AST and ALT were also measured.

Women with PCOS had a fivefold increased prevalence of liver injury as assessed by the levels of ALT and AST than women without PCOS. Furthermore, after adjustment for BMI, CK-18 fragment levels were significantly increased, indicative of elevated hepatocyte apoptosis, in patients with PCOS compared with women without PCOS. Among patients with PCOS, although metabolic phenotypes such as cholesterol levels and BMI significantly correlated with CK-18 fragment level, only hyperinsulinemia (measured by area under the curve of insulin response) was an independent predictor of CK-18 fragment level. The researchers point out the important role of insulin resistance in the pathogenesis of NASH implied by this result. Alarmingly, 27.6\% of patients with PCOS had CK-18 fragment levels indicative of NASH; by contrast, only one women without PCOS had such levels. No correlation was found between CK-18 fragment levels and those of ALT or AST.

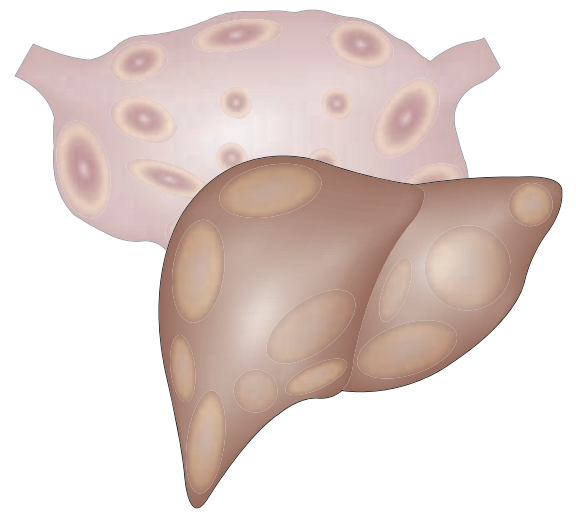

Lead investigator Ali Canbay of the University of Duisburg-Essen, Germany concludes that the findings have some important implications. "First, as abnormal liver enzymes did not predict CK-18 fragment elevation, evaluation of NASH in all patients with PCOS should be performed independently of liver enzyme levels. Indeed, we have previously shown fibrosis stage of 2 or higher in obese patients, although levels of liver enzymes mostly stayed in the reference ranges or were only slightly elevated. Secondly, the prevention of NASH and its long-term consequences represents a new but imperative therapeutic target in the treatment of patients with PCOS. Lastly, apoptotic cell death index may serve as a novel noninvasive method to evaluate NASH in PCOS."

\section{Carol Wilson}

Original article Tan, S. et al. Apoptotic markers indicate nonalcoholic steatohepatitis in polycystic ovary syndrome. J. Clin. Endocrinol. Metab. 95, 343-348 (2009) 